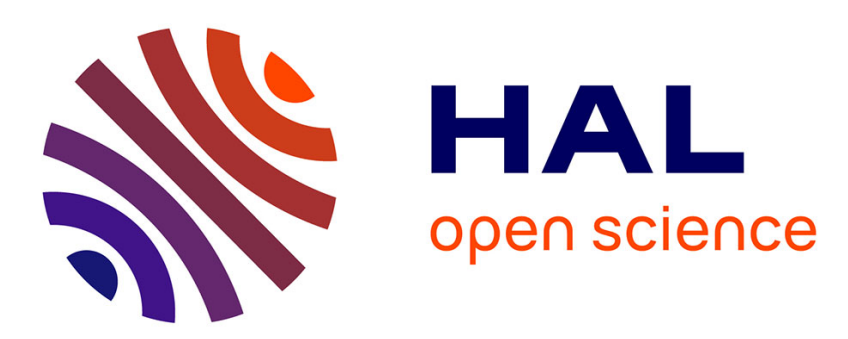

\title{
Large eddy simulations and stereoscopic particle image velocimetry measurements in a scraped heat exchanger crystallizer geometry
}

Marcos Rodriguez, Florent Ravelet, Rene Delfos, J. J. Derksen, Geert-Jan Witkamp

\section{To cite this version:}

Marcos Rodriguez, Florent Ravelet, Rene Delfos, J. J. Derksen, Geert-Jan Witkamp. Large eddy simulations and stereoscopic particle image velocimetry measurements in a scraped heat exchanger crystallizer geometry. Chemical Engineering Science, 2009, 64 (9), pp.2127-2135. 10.1016/j.ces.2009.01.034 . hal-00375870

\section{HAL Id: hal-00375870 https://hal.science/hal-00375870}

Submitted on 16 Apr 2009

HAL is a multi-disciplinary open access archive for the deposit and dissemination of scientific research documents, whether they are published or not. The documents may come from teaching and research institutions in France or abroad, or from public or private research centers.
L'archive ouverte pluridisciplinaire HAL, est destinée au dépôt et à la diffusion de documents scientifiques de niveau recherche, publiés ou non, émanant des établissements d'enseignement et de recherche français ou étrangers, des laboratoires publics ou privés. 


\title{
Large Eddy Simulations and stereoscopic particle image velocimetry measurements in a scraped heat exchanger crystallizer
}

M. Rodriguez Pascual ${ }^{1 *}$, F. Ravelet ${ }^{2}$, R. Delfos ${ }^{1}$, J.J. Derksen ${ }^{3}$, G.J. Witkamp ${ }^{1}$

${ }^{1}$ Process \& Energy Dept. Delft University of Technology, the Netherlands

${ }^{2}$ Institut de Mécanique des Fluides de Toulouse, France

${ }^{3}$ Chemical \& Materials Engineering Dept., University of Alberta, Canada

*Corresponding author: M.Rodriguez@tudelft.nl

\begin{abstract}
The transport phenomena in scraped heat exchanger crystallizers are critical for the process performance. Fluid flow and turbulence close to the heat exchanger (HE) surface as generated by stirring elements and scraper blades are crucial in this respect as they aim at avoiding an insulating scale layer on the HE surface. For this reason we performed largeeddy simulations of the turbulent flow (at a Reynolds number of $5 \cdot 10^{4}$ ) in a typical cooling crystallizer geometry with a focus on the bottom region where the heat exchanging surface was located. The simulations were validated with stereoscopic PIV experiments performed higher up in the crystallizer. For reasons of optical accessibility being hindered by the scrapers, the experiments could not be done near the heat exchanging surface. The flow structures as revealed by the large-eddy simulations could explain the local occurrence of scaling on an evenly cooled heat exchanger surface, and its irreproducibility caused by instantaneous cold spots.
\end{abstract}

Keywords: Fluid Mechanics, Crystallisation, Scaling, Heat transfer, Lattice Boltzmann Method, Scraped Heat Exchanger Crystallizers. 


\section{Introduction}

In several types of cooling crystallization processes, solutions or melts are brought by the action of heat exchangers into supersaturated regions where crystals will form. In industrial continuous processes, where solution is constantly fed into the crystallizer, control and stability of the bulk solution temperature is mandatory. For this reason a degree of turbulence inside the crystallizer is necessary to achieve good mixing of the whole solution, and with this aim stirrers are commonly used. Several computational and experimental studies of fluid flow in stirred tanks have been done in the past with a view to optimizing mixing processes motivated by their general importance in the chemical process industries [Yianneskis et al.,(1987) ; Schäfer et al., (1998); Derksen et al., (1999) ; Derksen and Van den Akker, (1999)]. In cooling crystallization this turbulent mixing determines the heat transfer rates that are directly responsible for the production rates, while the residence time in the crystallizer determines size and quality of the crystals. Particle flow interactions relate to solid separation, attrition and agglomeration processes. Studies on the influence of the flow on the heat and mass transfer and particle-flow interactions are of major interest but not an easy task to perform due to the complexities related to turbulence and the wide range of length and time-scales involved.

To bring the solution to the desired supersaturation heat exchangers (HE's) are typically in direct contact with the solution. The thermal boundary layer near the HE surface depends on the crystallizer flow characteristics. Because of the higher supersaturation at the HE surface the nucleation and growth rates of the crystals are faster than in the bulk of the 
solution. This situation is responsible for the formation of a scale layer of crystals on the HE surface in several cases of cooling crystallisation from a solution and (in particular) from a melt, including ice crystallisation from aqueous solutions. This scale layer reduces heat transfer, thus affecting the stability and efficiency of the crystallization process. To avoid this scale layer formation, it is common to use mechanical actions such as scraping. Depending on the composition of the solution and the characteristics of the mechanical scraping action (velocity, scraper shape and applied force), a maximum temperature difference between the solution and the heat exchanger surface can be maintained without the occurrence of scaling. In any case, to prevent scaling the heat exchanger surface temperature also has to be as uniform as possible, avoiding cold spots where scaling will start.

A laboratory scale scraped heat exchanger crystallizer was specifically designed to study the ice scaling behaviour of aqueous electrolyte solutions (Figure1). Further details of the setup and operation of this crystalliser can be found in [Rodriguez Pascual et al.(2008); Vassen et al.(2002); Pronk et al.(2005)].

\section{Figure 1}


The location where the scaling starts as well as the lack of reproducibility and spread in experimental results encountered in previous studies prompted us to investigate the local temperature and heat transfer distribution across the heat exchanger (HE) surface. These HE surface temperatures were measured by using a thermo chromatic liquid crystal sheet (TLC) placed directly upon the HE-surface (Figure 2). Further details of the experimental procedure are explained in Rodriguez Pascual et al.(2008).

\section{Figure 2}

The result shows that, even though the process as such is stationary, the HE surface temperature is far from uniform. Near the shaft and close to the wall the heat transfer between $\mathrm{HE}$ and solution, reflected by the calculated heat transfer coefficient $h_{c}(r)$, is lower by a factor of at least five than halfway the radius. So the question why $h_{c}(r)$ varies so much over the scraped HE plate had to be answered. Since the heating of the HE plate did not show local variations, the differences in local heat transfer could only be caused by the flow field. The accessible part of the flow field above the scrapers and below the stirrer was therefore investigated using Stereoscopic Particle Image Velocimetry, (3C-PIV); further details can be found in [Rodriguez Pascual et al.(2008)]. The axial, radial and tangential velocities of the flow field in this middle section of the crystalliser were derived from these PIV images.

It was, however, not feasible to measure the velocities in the lower scraper region just above the HE surface where the actual scaling occurs because the revolving scrapers hindered the optical accessibility required for PIV. For a more 
detailed analysis of the flow field in this region we were obliged to rely on information obtained by modelling the flow field.

To model the flow large eddy simulation (LES) in a lattice-Boltzmann scheme for discretizing the Navier-Stokes equations was used. The major reasons for employing a lattice Boltzmann discretization scheme are its almost full locality of operations, its computational efficiency (in terms of floating-point operations per lattice site and time step), and its ability to simulate flows in complex geometries. An interface model to represent the revolving parts of the geometry was avoided by modelling of the moving geometry with an adaptive force field technique [Derksen \& Van den Akker (1999)].

The LES methodology has proven to be a powerful tool to study and visualize stirred tank flows, because it accounts for the unsteady and periodic behaviour of these flows, and it can effectively be employed to explicitly resolve phenomena directly related to the unsteady boundaries. Results of the single-phase LatticeBoltzmann LES code applied to stirred tank flow have been extensively compared to phase-averaged and phase-resolved experimental data [Derksen \& Van den Akker (1999); Derksen (2001)].

The computational results of the flow field for the scraped heat exchanger crystalliser geometry used in this study are presented in this paper. To validate the correctness of the calculated flow field characteristics the results for the middle section of the crystalliser were compared with the previous experimental results from the 3C-PIV measurements.

\section{Simulation setup}


The Navier-Stokes equations governing the flow in the stirred tank were discretized according to the lattice-Boltzmann method. This is an inherently parallel and efficient numerical scheme for computational transport physics (see e.g. Succi (2001) for an overview). The strong turbulence generated by the impeller and scrapers prohibits direct numerical simulation: the computational effort for resolving all length and time-scales would be too large.

In large-eddy simulations only the large-scales are resolved explicitly - the rationale behind it is that the small scales behave more universally and are therefore more prone to modelling than the large scales (Lesieur \& Métais (1996)). Modelling instead of resolving the small scales alleviates the computational burden. For modelling of the small scales the standard Smagorinsky subgrid scale model was applied. A value of $c_{S}=0.12$ was adopted as the Smagorinsky constant, which is within the range of values commonly used in shear-driven turbulence.

The stirred-scraped crystallizer configuration consists of a cylindrical, flatbottomed, tank with diameter of $240 \mathrm{~mm}$ and a height of $300 \mathrm{~mm}$. The flat bottom area of the crystallizer that acts as the heat-exchanging surface is scraped by a set of four rotating scraper blades with a diameter of $198 \mathrm{~mm}$ that is driven by a vertical shaft. Halfway the shaft a $45^{\circ}$ pitch blade turbine with a diameter of $100 \mathrm{~mm}$ is installed to keep the slurry homogeneously mixed. The geometry is defined in figure 3a. The geometry of the scraper in the simulations closely mimics the experimental geometry (Figure 3b and 3c).

The uniform, cubic grid inherent to most of the lattice-Boltzmann formulations comprised $1.8 \cdot 10^{7}$ nodes. In terms of spatial resolution: the linear size of a cubic 
lattice cell amounted to approximately $1 \mathrm{~mm}$. The time step in the LES was such that one impeller revolution took 4200 time steps.

The Reynolds number that fully determines this single-phase flow is defined as $\mathrm{Re}=N D^{2} / v$ with $N$ the impeller speed in rps, $D$ the diameter of the scraper (being 198 $\mathrm{mm}$ ) and $v$ the kinematic viscosity of the working fluid. The continuous phase was water with a density $=10^{3} \mathrm{Kg} / \mathrm{m}^{3}$ and $v=10^{-6} \mathrm{~m}^{2} / \mathrm{s}$. The scraper and impeller were set to rotate at a speed of $77 \mathrm{rpm}$ corresponding with $\mathrm{Re}=5 \cdot 10^{4}$.

The LES is started from a zero velocity field. In the start-up phase of the flow we monitor the total kinetic energy in the tank. Once this has stabilized we start collecting flow data for later statistical analysis such as average velocity and turbulent kinetic energy fields. .

\section{Figure 3}

\section{Results and discussion}

\subsection{The overall flow field in the crystalliser}

The flow field as measured earlier by 3C-PIV for the middle section of the crystallizer qualitatively explains the heat transfer distribution at the HE-surface as obtained from the (TLC) measured surface temperatures. In the core around the shaft the heat transfer is low because of the absence of radial and axial flow [Rodriguez Pascual (2008)]. From about $50 \mathrm{~mm}$ from the shaft a secondary flow descends onto the HE surface, and causes a much better heat transfer in the middle of the HE surface. In contrast the local heat transfer is reduced in the outer ring of the $\mathrm{HE}$ surface where the fluid temperature on its way to the wall has adapted the bottom temperature before it ascends at the wall (Figure 4). 


\section{Figure 4}

In Figure 5 the dash-dotted line represents the tangential velocity of the scraper. The solid line representing the tangential flow measured by the PIV, confirms that the solution is in solid body rotation up to about $40 \mathrm{~mm}$ from the shaft. This unwanted behaviour of the flow prevents proper mixing of the rotating solution with the bulk, and causes low heat transfer at the bottom of this region. When moving radially inward or outward, the flow tends to conserve its angular momentum; in a frictionless flow this may lead to a vortex. As a simplified physical model of the flow, we fitted the measured tangential velocity profile with the Oseen vortex solution [Batchelor(1967)], which gradually transits from a solid-body rotation in the inner region to a free vortex flow in the outer the region. The transition takes place at approximately $r=40 \mathrm{~mm}$, as shown in Figure 5 . The measured tangential flow matches qualitatively with the vortex flow in the Oseen model.

\section{Figure 5}

Although we now can roughly explain the temperature profile at the HE surface, for a more detailed explanation the flow pattern between the scrapers just above the surface has to be studied. Due to the problematic measuring access created by the presence of the rotating scrapers we have for this purpose to rely on CFD simulations. Figure 6a shows the axial and radial time averaged velocities for the whole tank as obtained from LES computational simulations, under steady-state total kinetic energy condition, plotted as vectors with normalized lengths. To validate the computational results we compare the simulated flow velocities with the velocities calculated from the 3C-PIV measurements in the same middle region of the 
crystalliser. This region is indicated in Figure $6 a$ by a black rectangle at the right hand side of the picture.

\section{Figure 6}

Zooming in on the simulations of the flow velocities in the PIV measured area in figure $6 \mathrm{~b}$ demonstrates that also in the simulations axial or radial flow is nearly non existent near the shaft. The middle area shows a strong axial flow towards the bottom with a non existent radial contribution. Close to the outside wall the flow is carried upwards with also no radial contribution, so these results are in good agreement with the stereoscopic PIV data in Figure 4. The flow velocities at the three lines across the measured area at three different heights of 45, 59 and $75 \mathrm{~mm}$ from the bottom plate are presented in figure 7.

\section{Figure 7}

The tangential and axial velocities at the three heights are plotted against those calculated from the stereoscopic PIV experiments. The results match quite well, although the tangential and axial velocities from the PIV experiments are slightly smaller than the LES values. Also the tangential velocities decrease when approaching the outside wall, while the LES values increase slightly before decreasing at the wall. The agreement is however in general very satisfactory, and the observed differences can be explained by the unavoidable inaccuracies in the experimental results and by the idealised computational approach.

A qualitative comparison between the PIV and LES snapshots of the same portion of the flow (Figures 8 and 9, drawn with the same scale for the velocity vector length) shows interesting resemblance. Clearly turbulence is much stronger 
close to the outer tank wall. Furthermore the sizes of the turbulent structures observed in PIV and LES agree quite well.

\section{Figures $8 \& 9$}

\subsection{Flow field at the bottom part between the scrapers.}

Now that we have confidence in the simulated flow data through their agreement with the PIV results, the non accessible area just above the scraped heat exchanger surface can be analysed from the LES simulations.

By considering time averaged flow fields resolved for the angular position of the scraper in the LES more information about the flow patterns near the bottom can be gained. In Figure 10 we show the average flow one in a vertical cross section through the scrapers (Figure 10a) and one in between the scraper blades (Figure 10b).

\section{Figure 10}

The cross-section through the scrapers (Figure 10a) shows that the solution at the tip of the scraper is forced outwards to the wall and subsequently upwards. Another important observation is that in the middle region the downward flow above the scraper is carried radially towards the centre of the crystalliser instead of being carried outwards as happens in between scrapers (Figure10b).

To be able to explain the radial variation in local heat transfer, a more detailed analysis of the region between the scrapers is needed. Figure 11 zooms in on the time averaged flow in this area half-way between the scrapers, in the region from the shaft to the wall on the horizontal axis and from the 3C-PIV measured area down to the scraped HE surface on the vertical axis. 


\section{Figure 11}

In the scraper region $\mathrm{z}=0$ to $35 \mathrm{~mm}$ height from the HE surface, from the shaft till more or less $\mathrm{r}=10 \mathrm{~mm}$ in the radial direction the solution has hardly an axial or radial velocity component. Between $\mathrm{r}=10$ and $15 \mathrm{~mm}$ a vertical down flow is observed but with no radial component explaining the low heat transfer because limited mixing with the rest of the tank volume. Beyond $\mathrm{r}=15 \mathrm{~mm}$ the liquid starts getting axial and radial velocity components, mainly towards the outside tank wall. If we look onto the high transfer region in Figure 11 between $r=15$ and $62 \mathrm{~mm}$, we can observe that this is the area where the axial velocity is positive removing the liquid from the heat exchanger surface upwards. Here we actually have to differentiate between two levels: the fluid trajectories starting between $\mathrm{r}=15 \mathrm{~mm}$ and $40 \mathrm{~mm}$ from the shaft (represented by the top streamline in Figure 11) and the fluid trajectories starting between $\mathrm{r}=40 \mathrm{~mm}$ and $60 \mathrm{~mm}$ (bottom curve in Figure11). As we can see, the top trajectory brings the liquid higher up after which it is carried into the main secondary flow that transports the fluid to the top of the crystalliser. In the bottom fluid trajectory the axial velocity is lower. This keeps the liquid close to the heat exchanger surface while approaching the outside tank wall. This makes that the fluid in the bottom trajectory is being cooled further on its way along the trajectory. This limits the local heat transfer coefficient. This could explain why from $r=40 \mathrm{~mm}$ on the TLC measurements showed that the local heat transfer coefficient starts to decrease [Rodriguez Pascual (2008)]. Also note that fluid can get trapped by the vortex formed at the scraper tip at the HE surface from $r=100 \mathrm{~mm}$ until the outside wall. This eddy retains the liquid in this region rotating without much mixing with the rest of the liquid causing even lower local heat transfer close to the wall. Also very 
close to the bottom a small inward radial flow is present in between the scrapers (Figure 11); in Figure 10a a small radial flow is pushed outwards by the action of the scraper. The net effect is quite important as it explains the shape of the region of high heat transfer between two scraper passages as shown in the TLC measurements [Rodriguez Pascual (2008)]. This region becomes less wide when the next scraper approaches. The fluid descending from the bulk to the HE surface by the scraper action is thus pushed outwards to the wall. Some of this fluid is captured by the lower eddy close to the wall, and redirected to the shaft, until the next passing scraper sends it back again to the wall. This redirected fluid is colder and narrows the area of higher heat transfer.

\section{Figure 12}

For a better understanding, we should not forget that the three directional components of the flow are together responsible of the complete flow field and its influence in the local heat transfer. Figure 12 shows the time-averaged tangential, radial and axial velocities at heights of $z=5,15$ and $30 \mathrm{~mm}$ from the bottom in the scraper region. Here we can see how the tangential velocity is dominating throughout all radial positions and that the liquid is largely undergoing solid body rotation. It is remarkable that the area where the heat transfer is relatively high (between $\mathrm{r}=15$ and $62 \mathrm{~mm}$ ) axial and radial velocities are quite low, and we would have missed the effect if we had not looked at the vertical cross sections. We can however, still appreciate from figure 12 how the radial component increases from the bottom and decreases again when approaching the top of the scraper.

So far we only considered time-averaged velocities and related flow structures in relation to heat transfer. This is a valid approach given the relatively long response 
times of the HE surface temperature. However, eventually instantaneous turbulent flow is responsible for the local, time-dependent heat transfer. This implies that time dependent cold spots could be formed where nucleation starts. We therefore now turn to instantaneous realizations of the flow field as qualitative indicators for heat transfer.

To do that we first image a horizontal cross section of the crystalliser flow at a level halfway the height of the scrapers (Figure 13). This cross section is in the frame of reference of the rotating scraper. It reveals that the scraper tip creates a turbulent flow near the wall behind its blade that dissipates its energy quite rapidly (over roughly half the distance between two scraper blades). The liquid near the tank's outer wall does not mix very well with the rest; it largely flows along the wall until the next scraper blade comes by. The instantaneous differences in local turbulence could however, provoke instantaneous cold spots. At these spots nucleation can start, which is a statistical and instantaneous event followed by fast lateral growth. This would explain the lack of reproducibility and spread in scaling experiments. Near the shaft the flow is less erratic and no instantaneous cold spots are expected there.

\section{Figure 13}

The high heat transfer in the middle area of the HE surface is still difficult to interpret from Figure 13. For this purpose vertical cross section of the flow was made perpendicular to the scraper blade at $r=60 \mathrm{~mm}$ from the shaft, see Figure 14 .

\section{Figure 14}

This cross section shows how in the middle of a scraper blade the liquid from the bulk is carried down to the HE surface in near the wake of the scraper. From figure 11 we now know that this flow at the back of the scraper is pushed outwards 
to the outside wall and that later on (in between the two scrapers) it is moved upwards away from the HE surface in the region from around $15 \mathrm{~mm}$ until $40 \mathrm{~mm}$. Therefore the contact time of the liquid with the heat exchanger surface in the middle region of a scraper blade is lower than in the other areas, and because the liquid comes directly from the bulk, the temperature difference compared with the cooling liquid underneath the HE surface is higher. This down flow also reduces the thickness of the thermal boundary layer, which enhances the heat transfer. At the front of the scraper the liquid is pushed upwards, and most of it is moved outwards and merges with the main secondary flow that runs to the top of the crystalliser.

Realizing that the main cause responsible for the inhomogeneous distribution of local heat transfer on the HE surface is the axial velocity component we now consider the instantaneous axial velocity at three horizontal cross sections at heights of 5,15 , and $30 \mathrm{~mm}$ (Figure 15).

\section{Figure 15}

As we can see from Figure 15 the fluid is brought down to the heat exchanger in the wake of the scraper blades. This downward axial velocity behind the scraper is zero near the centre of the tank and increases (negatively) in the radial direction until close to the tip of the scraper where it turns positive as approaching the outside wall. As the height increases from 5 to $30 \mathrm{~mm}$ the downward velocity gets stronger. This figure also shows how the down flow coming from the bulk (as we already saw in Figure 14) is pushed up again by the front of the next scraper, and as we know from Figure 11 also outwards to the outside wall. This means that the scraper blade's middle region is continuously refreshed with every scraper passage by the bulk 
liquid, what explains the higher local heat transfer coefficient in the middle area of the HE surface compared to the centre and outside areas.

\section{Conclusions}

Cooling crystallization often suffers from scaling that starts at specific areas on a heat exchanger surface even if the HE surface is evenly cooled. In a previous paper this behaviour was investigated by measuring the local temperature distribution across a flat scraped HE surface located at the bottom of a stirred crystallizer.

These measurements showed that the temperature was not uniformly distributed with differences larger than $4{ }^{\circ} \mathrm{C}$. Such differences are sufficiently large to explain local variations in scaling tendency. After excluding all other possible reasons for this inhomogeneous distribution the fluid flow in the crystalliser seemed to be the only responsible cause. Stereoscopic PIV measurements of the flow in the region above the scrapers and below the stirrer were carried out, which showed that the flow pattern in this crystallizer geometry could roughly explain the observed inhomogeneities in heat transfer. For more detailed information on the flow pattern between the scrapers and thus more closely to the HE surface we had to rely on computational flow simulations, because this region was not accessible for PIV measurements.

The flow of the total scraped HE crystalliser was therefore computationally resolved by Large Eddy Simulations using a Lattice Boltzmann scheme. The simulations were validated by comparison with the PIV measurements performed previously. This provided sufficient confidence to focus on the flow pattern in the bottom region. 
Analysing the results from the LES we could explain the radial local heat transfer coefficient distribution along the HE surface. The local heat transfer is higher in the middle region of the HE surface between the shaft and the outer wall (from $r=$ 15 till $r=60 \mathrm{~mm}$ ) because the fluid comes directly from the bulk and its residence time near the HE surface is low. The lower local heat transfer coefficient in the tank's centre is because the fluid is largely in solid body rotation and although (when the scraper passes) liquid is carried up in front and down behind the scraper, it is not mixing in the radial direction with the rest of the liquid. Beyond $r=60 \mathrm{~mm}$ until around $\mathrm{r}=100 \mathrm{~mm}$, the local heat transfer coefficient is lower because the residence time of the liquid close to the HE surface is higher. In between $\mathrm{r}=100 \mathrm{~mm}$ and the outside wall, a worse situation for heat transfer is provoked by a static vortex that brings the fluid into a segregated area. These fluid flow results explain the mechanisms that provoke the local heat transfer distribution measured by the TLC experiments. Instantaneous fluctuations in the flow are too fast to have any impact on the temperature profile at the HE surface that depends on the different residence times of the fluid in the different areas.

The formation of an ice layer begins in the colder areas around the centre and near the outside wall of the HE surface, and spreads along the HE surface. In a clear fluid, scale formation starts by primary heterogeneous nucleation at the HE surface followed by a fast lateral growth rate. This nucleation is a statistical event, where local time-dependent cooling conditions play a role. Because the subsequent lateral growth is very fast, irreproducibility can be expected. Under crystallizing conditions crystals may also land on the HE surface. These crystals may firmly attach to the surface by growth, before they are removed by the scraper and contribute to the 
scale formation. This process also depends upon accidental events that make altogether the appearance of the ice scaling layer time dependent and difficult to predict.

The importance of the flow field for controlling the scale layer formation on the HE surface during crystallisation has been demonstrated. In the context of fluid flow, unexpected results found previously [Vaessen et al.(2002)]; Pronk et al.(2005)] where for example lower stirring/scraping velocities show less scaling formation may be understandable. Further computational analysis in this sense including solving of the local heat transfer and liquid temperature then has to be done.

Large eddy simulations using a lattice Boltzmann scheme have proven to be a powerful tool to investigate the flow in future complicated crystallizer designs with various scraper geometries, since inhomogeneities in heat transfer impose strong limitations on the performance of the process.

\section{Outlook}

The simulations presented here are those of the fluid dynamics of a clear liquid. Under crystallising conditions however, even without the occurrence of scaling, crystals that are developed in the bulk of the fluid may come close to or even land on the heat exchanger surface. These crystals are transported back into the bulk liquid by the flow induced by the revolving scrapers. In addition, under scaling conditions mostly small particles are scraped off the scale layer and lifted by the flow. The scraper design has a remarkable importance here and should be such that the particles are transported away from the HE surface by the flow; they should not directly return to the surface by e.g. a trailing vortex. The particles are subjected to 
collisions with the hardware such as the scrapers and the wall, and to particleparticle collisions. They may even agglomerate into lumps of separate or inter-grown particles. To properly simulate a flow which contains particles, first the particleparticle interactions have to be included. As a second more complicated step a coupled particle-flow interaction has to be implemented, because in areas of high particle densities these particles will affect the flow behaviour.

In a forthcoming paper a first step in this direction has been made by implementing the particle-particle interactions. Direct Numerical Simulations based on a Lattice Boltzmann scheme were carried out, and the results will be compared with an experimental visualisation of particles that are added to the fluid and that are transported by the scraper actions.

\section{Acknowledgment}

The authors would like to thank Prof. G. M. Van Rosmalen for in-depth discussions.

\section{References}

Batchelor, G.K.(1967) An Introduction to Fluid Dynamics. Cambridge University

Derksen, J. J.(1999), and H. E. A. Van den Akker. Large Eddy Simulations on the Flow Driven by a Rushton Turbine. AIChE J., 45(2),209.

Derksen, J.J., S. Doelman, and H.E.A. Van der Akker,(1999). Three dimensional LDA measurements in the impeller region of a turbulently stirred tank. Exp.Fluids,27,522. 
Derksen, J.J.(2001).Assessment of Large Eddy Simulations for Agitated Flows. Trans. IChemE, 79A, 824.

Lesieur, M., and O. Métais.(1996).New trends in large-eddy simulations of turbulence. Annu. Rev. Fluid Mech., 28, 45.

Pronk, P., Infante-Ferreira, C.A., Rodriguez Pascual, M. and Witkamp, G.J. (2005) Maximum Temperature difference without ice scaling in a scraped heat exchanger crystalliser.Proc. 16th Int. Symp. on Industrial Crystallization. pp. 1141-46

Rodriguez Pascual, M., Ravelet, F., Delfos, R., Witkamp, G.J.,(2008) Measurement of flow field and temperature distribution in a scraped heat exchanger crystallizer, Eurotherm, Eindhoven, May 18-22; also an extended version was submitted for publication in International Journal of Heat and Mass Transfer .

Schäfer, M, M.Yianneskis, P. Wächter, and F. Durst.(1998).Trailing vortices around a $45^{\circ}$ Pitched blade impeller. AICHE J.,44,1233.

Succi, S..(2001). The lattice Boltzmann equation for fluid dynamics and beyond, Clarondon Press, Oxford.

Vaessen, R.J.C., Himawan, C. and Witkamp, G.J. (2002), Scale formation of ice from electrolyte solutions on a scraped surface heat exchanger plate. Journal of Crystal Growth, 237-239 (Pt.3), pp. 2172-77 
Yianneskis, M.,Z. Popiolek, and J.H. Whitelaw(1987). An experimental study of the steady and unsteady flow characteristics of stirred reactors. J.Fluid Mech.,175,537. 


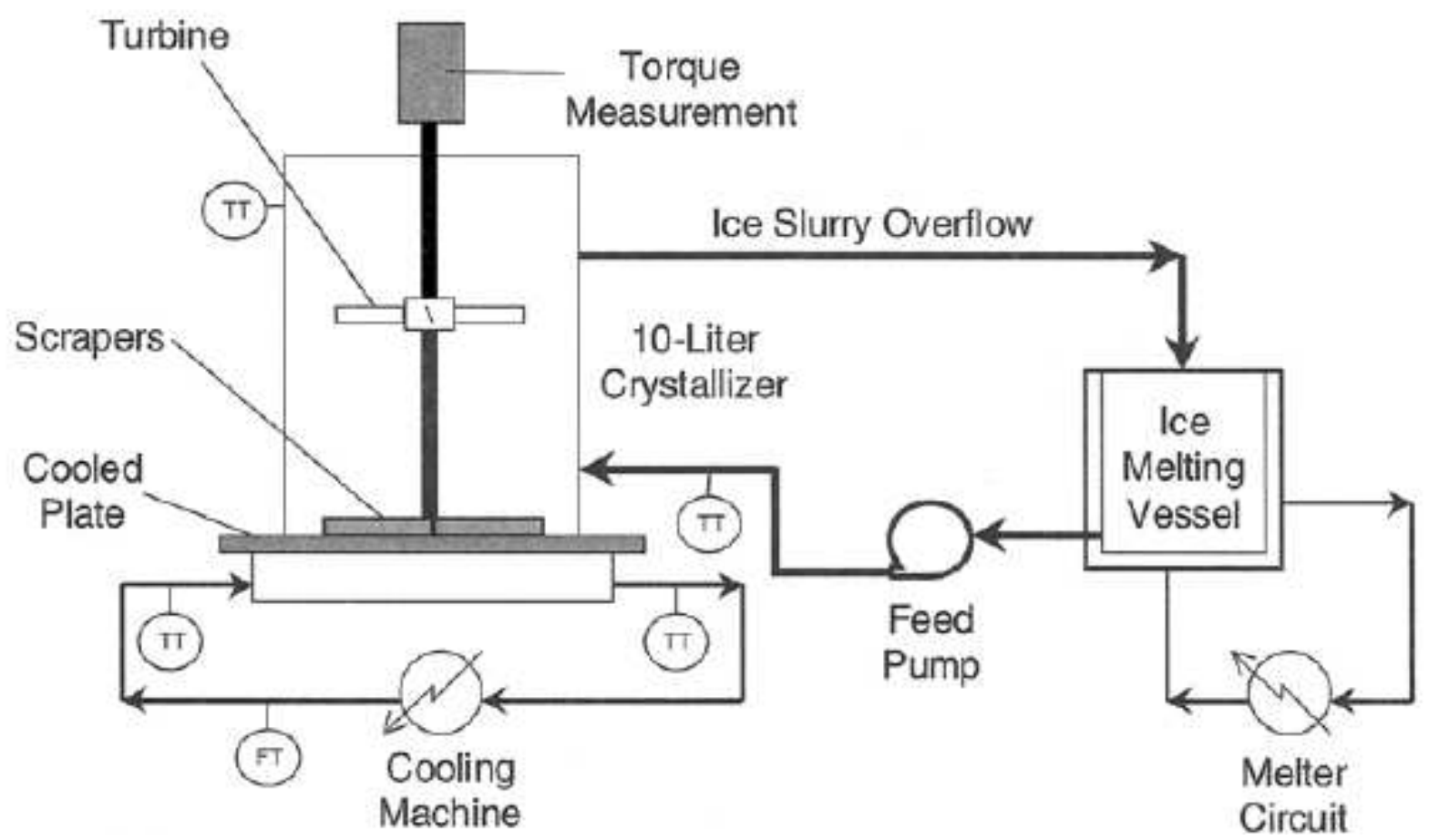

Figure1. Laboratory scale scraped cooling crystallizer. [Vassen et al.(2002)] 


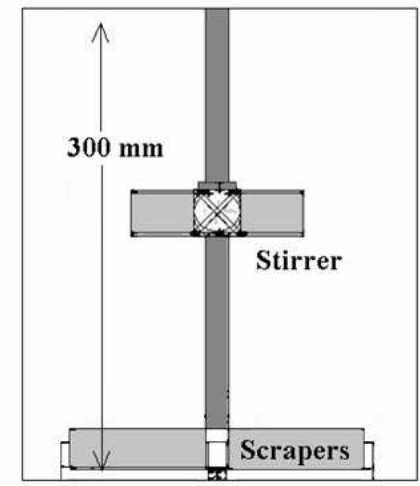

a)

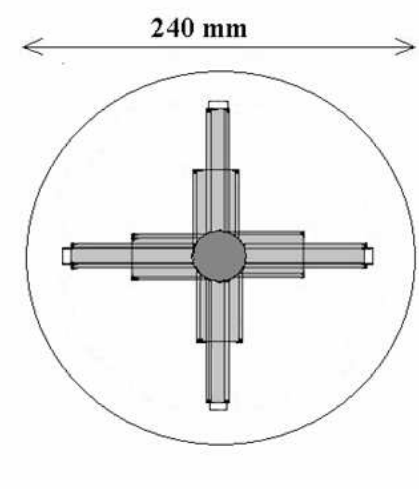

b)

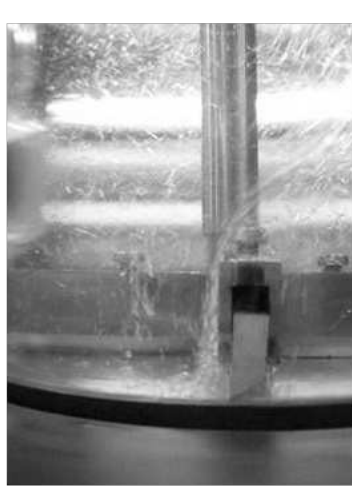

c)

Figure 3a). Side view of the crystalliser. b). Top view of the crystalliser. c). Sideway view of the real scraper geometry. 


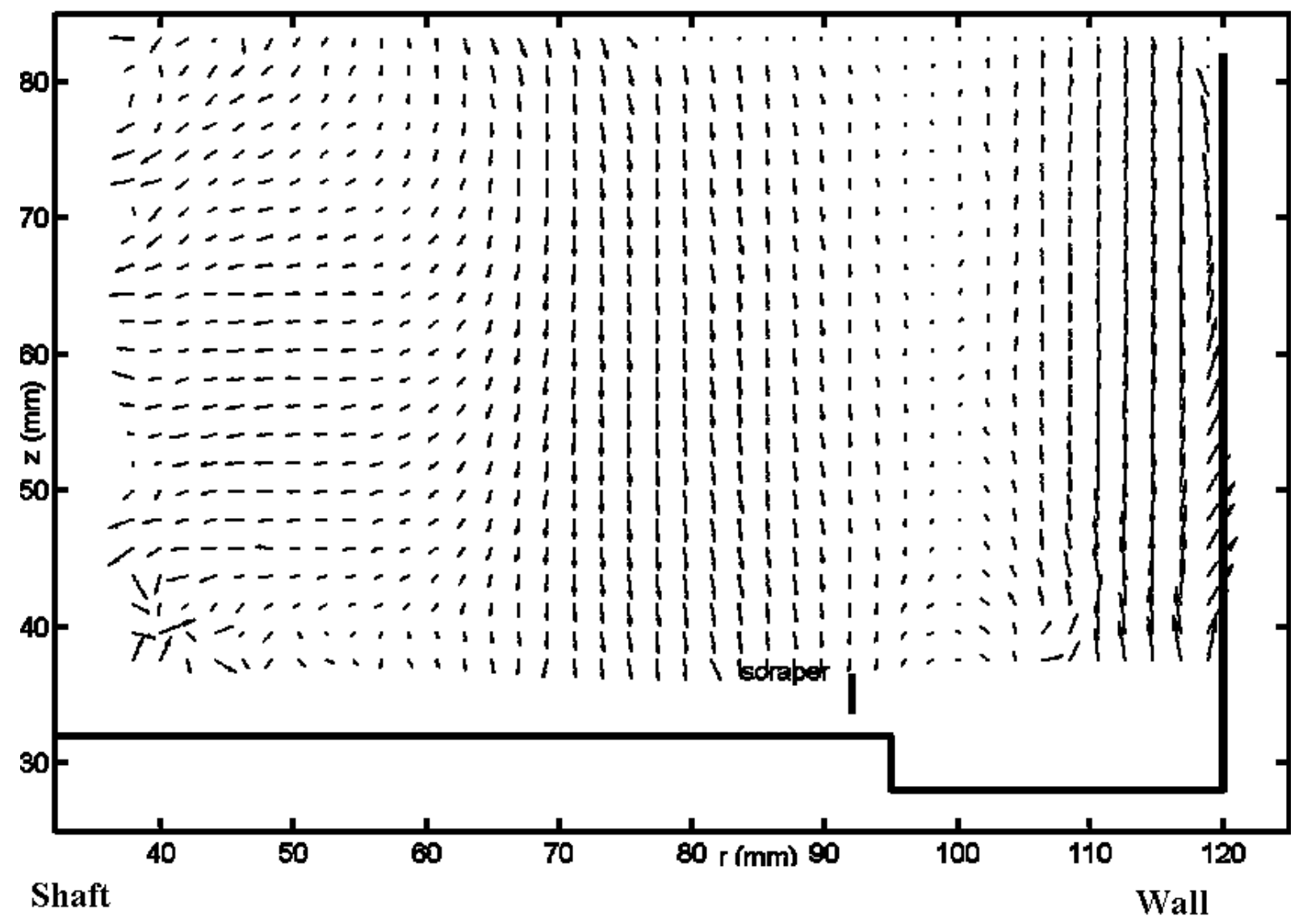

Figure 4. Time averaged radial and axial velocities measured by 3C-PIV depicted as vectors with their normalized length. 


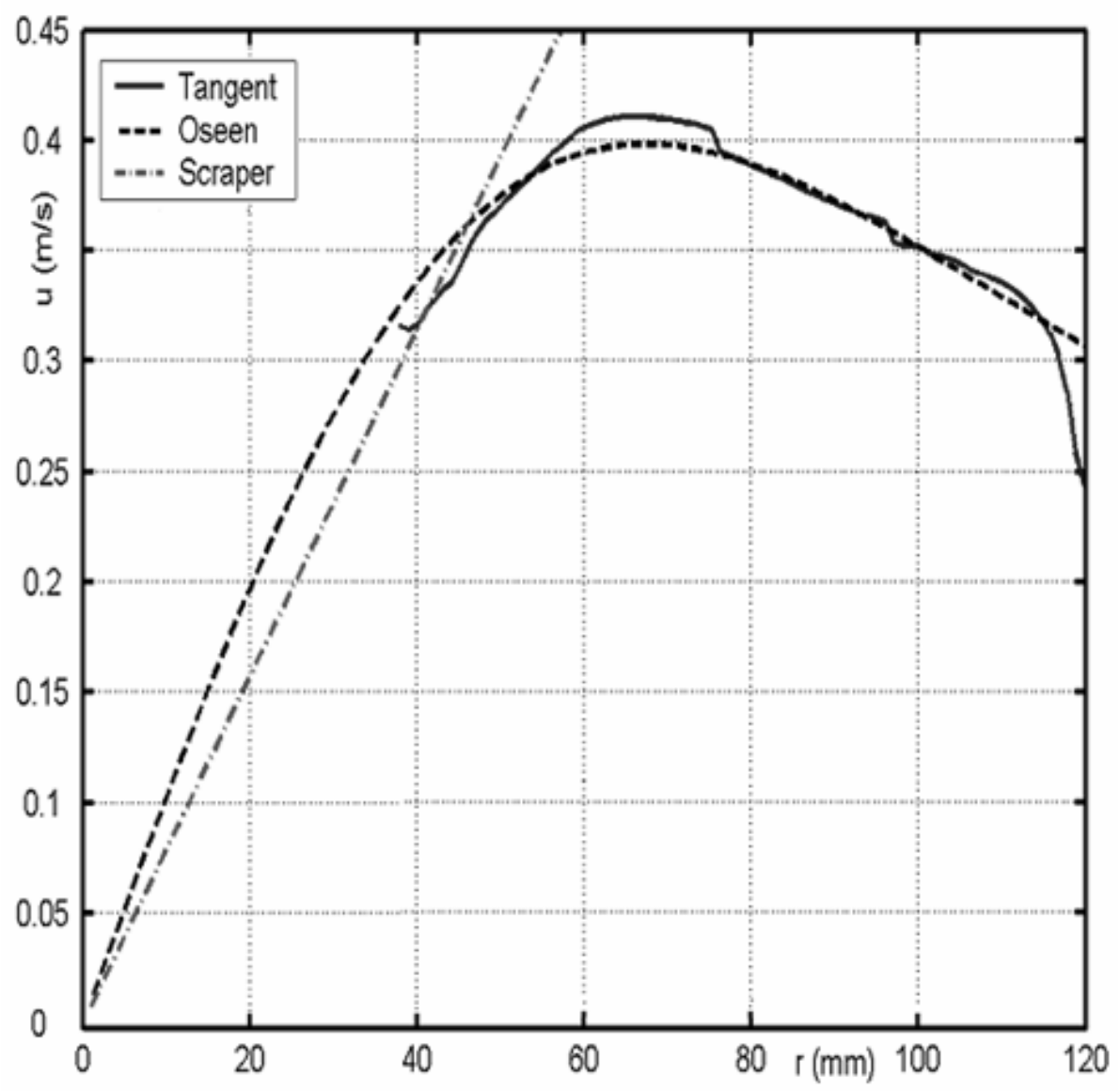

Figure 5. Measured time-axial averaged tangential velocity of the PIV area, the scraper velocity and the tangential velocity calculated from the Oseen approximation. 

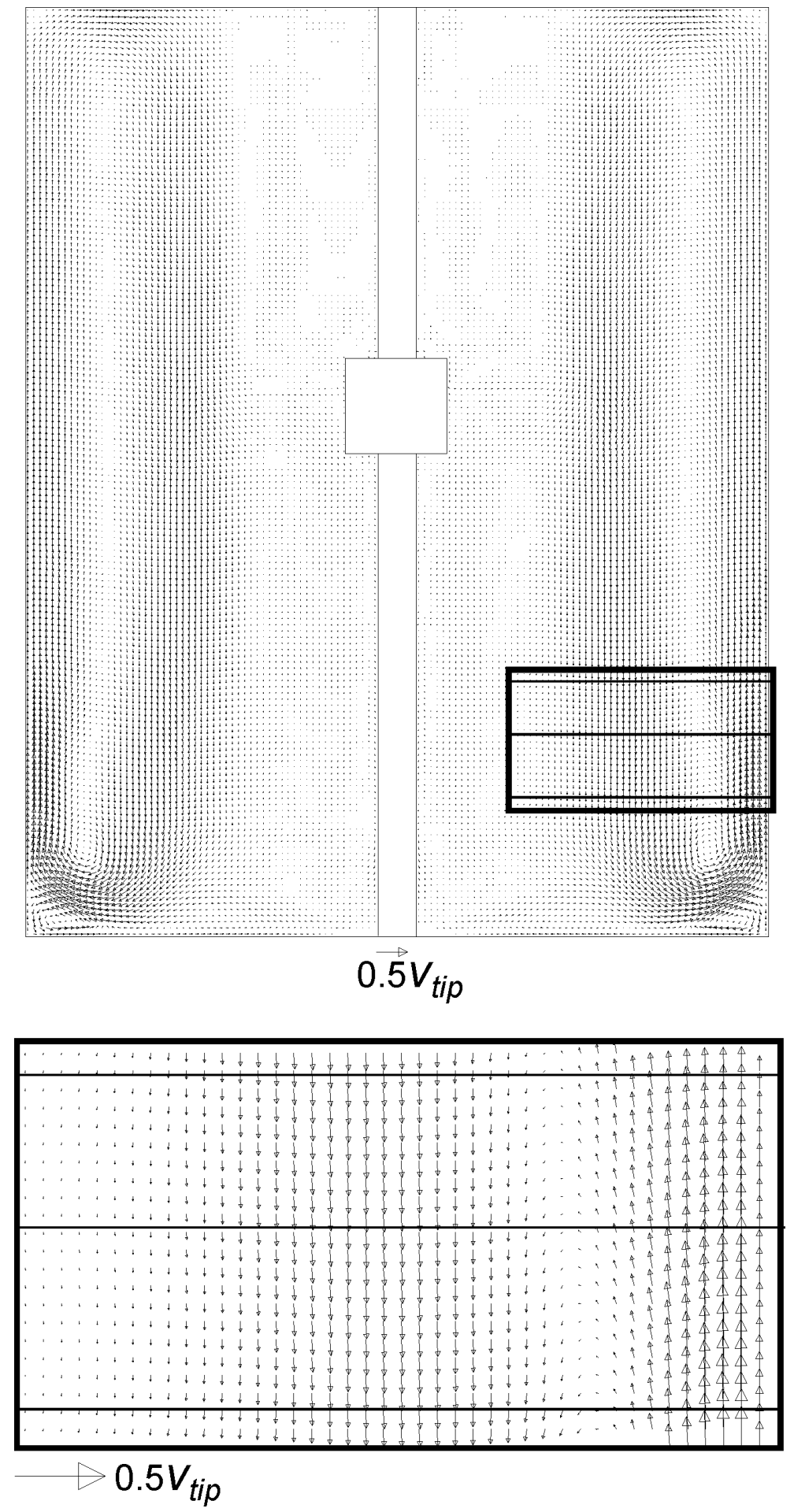

Figure 6. a) Time averaged velocity vectors of the flow field inside the scraped heat exchanger crystalliser. The black rectangle corresponds to the area measured by $3 \mathrm{C}$ PIV. b) Computational results of the 3C- PIV measured area. The three lines across the measured area are drawn at heights of 45,59 and $75 \mathrm{~mm}$ from the bottom plate. 


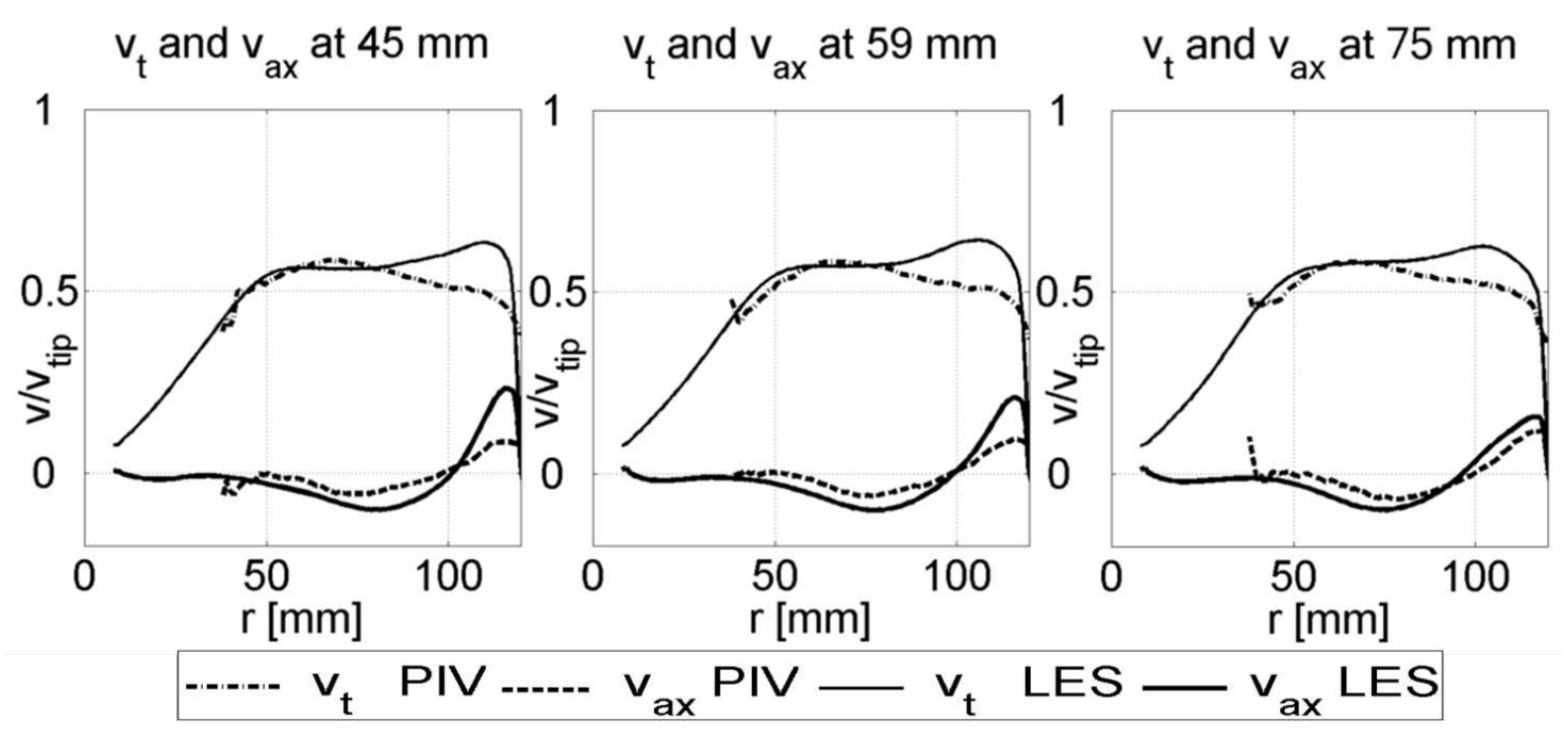

Figure 7. Tangential and axial velocities calculated from 3C-PIV experiments and from LES simulations at heights of 45, 59 and $75 \mathrm{~mm}$ from the bottom plate. 

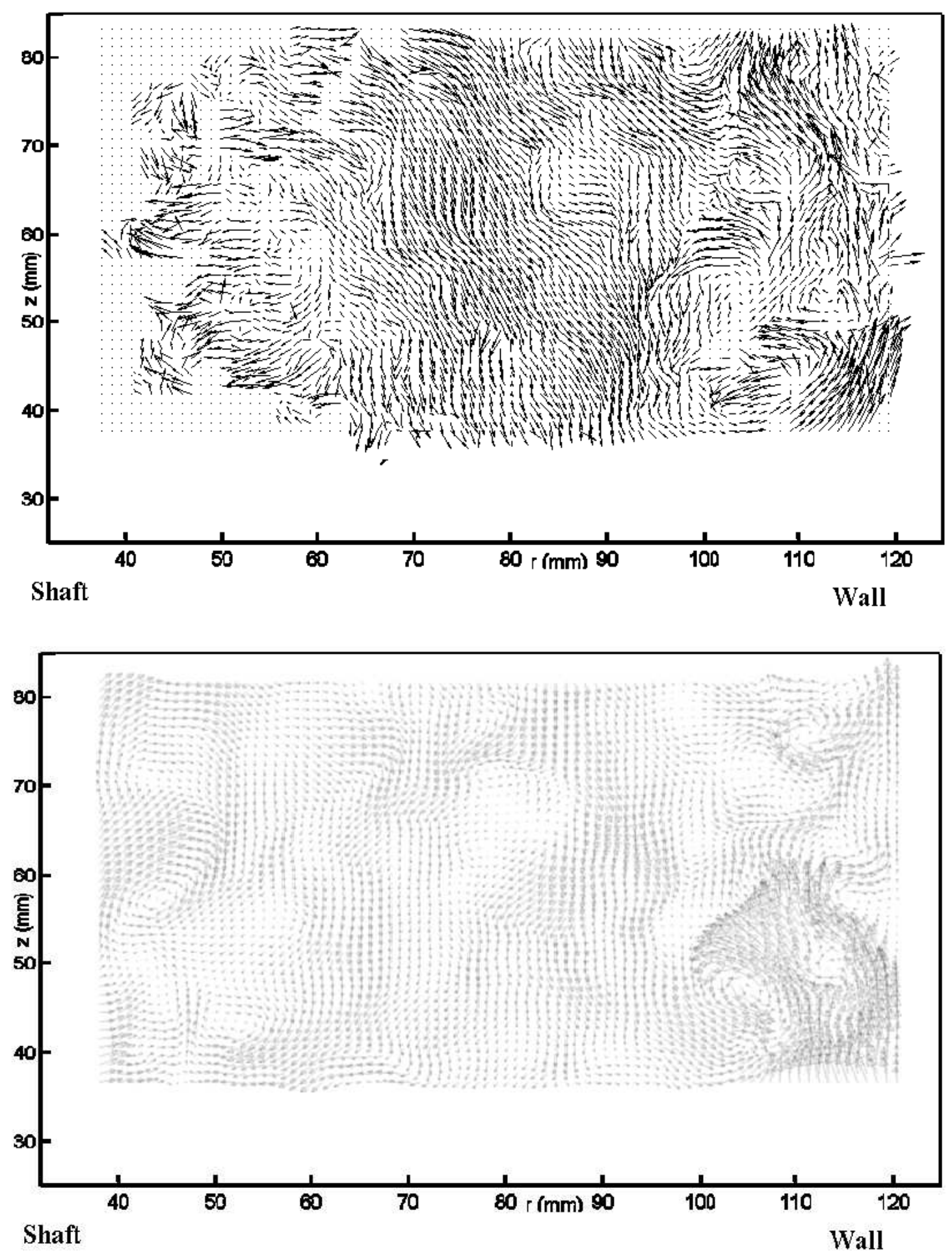

Figure 8 a). Instantaneous capture of the flow from the 3C-PIV measurements. Vectors represent the axial and radial velocity and their normalized length.b). Instantaneous capture of the flow from the LES simulations 

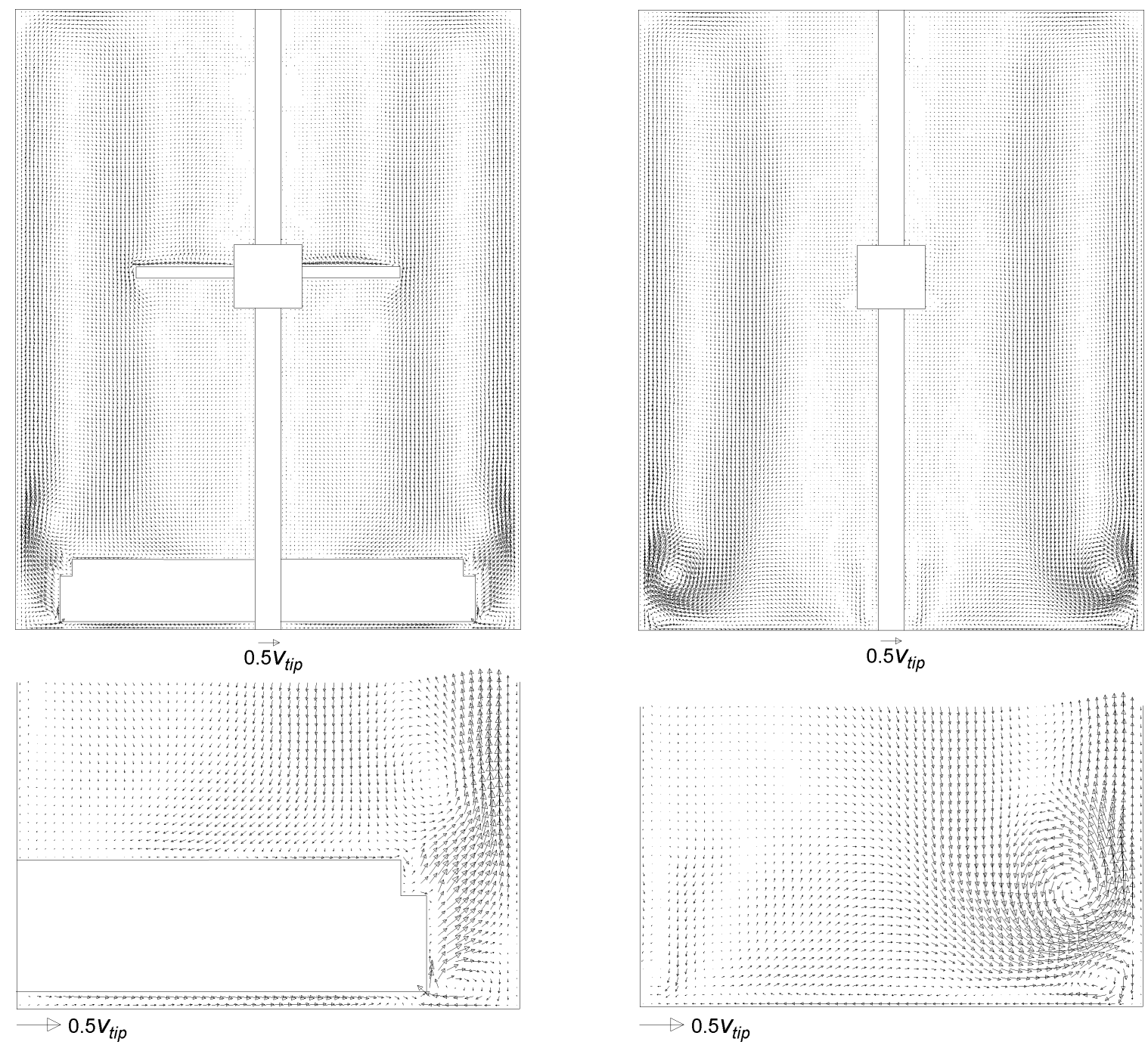

a)

b)

Figure 10. a) Time averaged flow field of the vertical cross section through the scrapers with an enlargement of the scraper area underneath. b) Time averaged flow field between the scrapers with an enlargement of the scraper area underneath. 


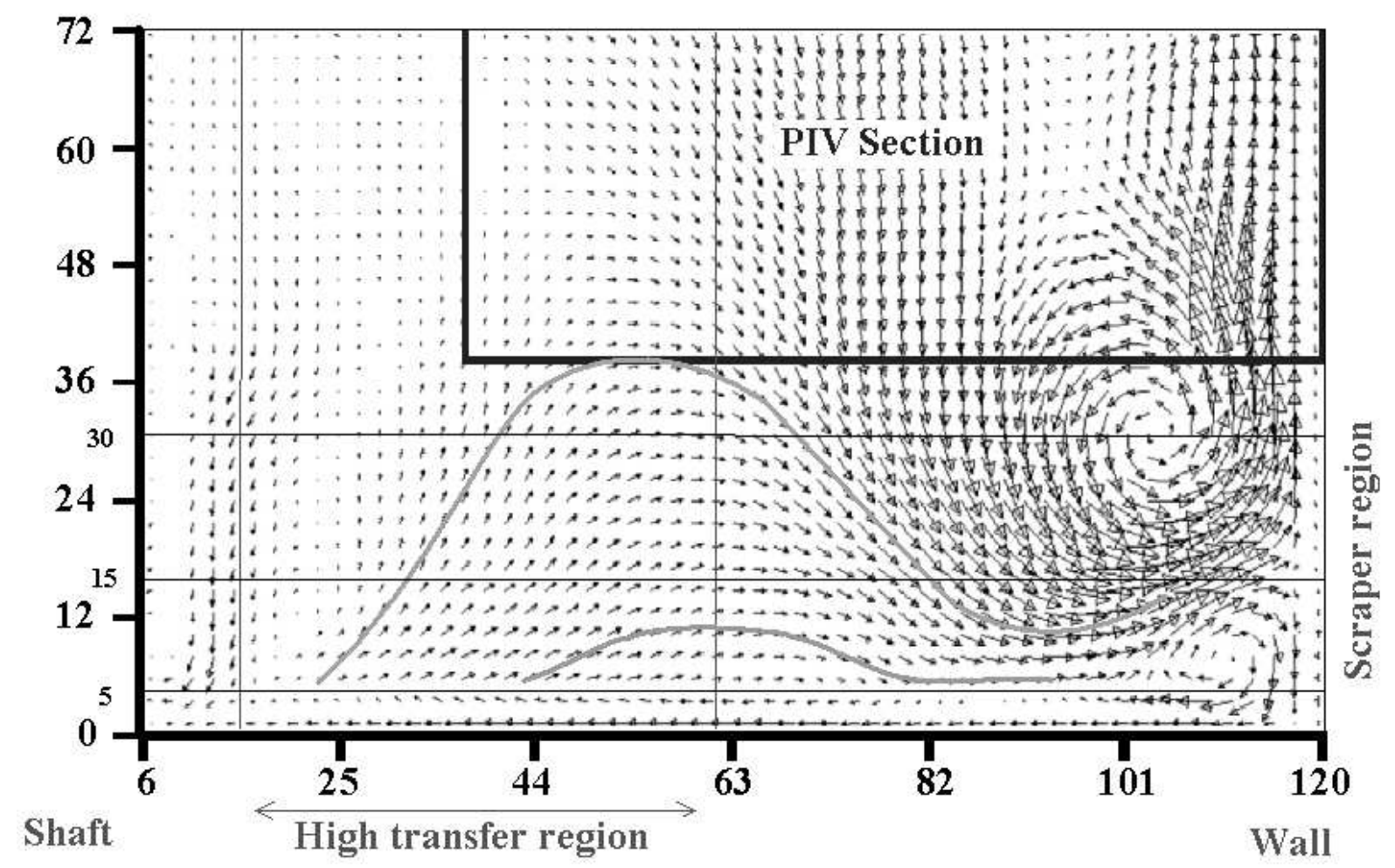

Figure 11. Axial-radial vector flow field area from the shaft to the wall and from the top of the 3C-PIV measured area to the scraped heat exchanger surface. 

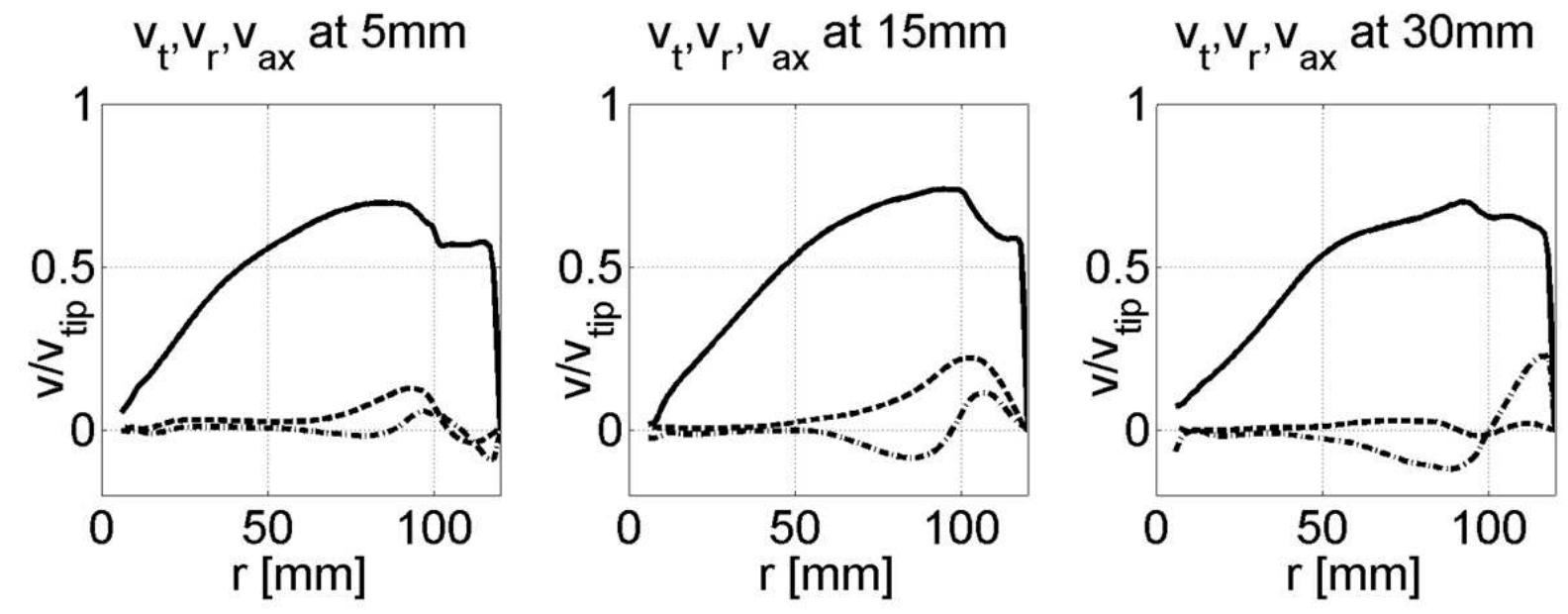

-...- $v_{\text {ax }}$ LES ---- $v_{r}$ LES - $v_{t}$ LES

Figure 12. Time averaged tangential, radial and axial velocities calculated from LES simulations at heights of 5,15 and $30 \mathrm{~mm}$ from the bottom plate in the scraper region. 

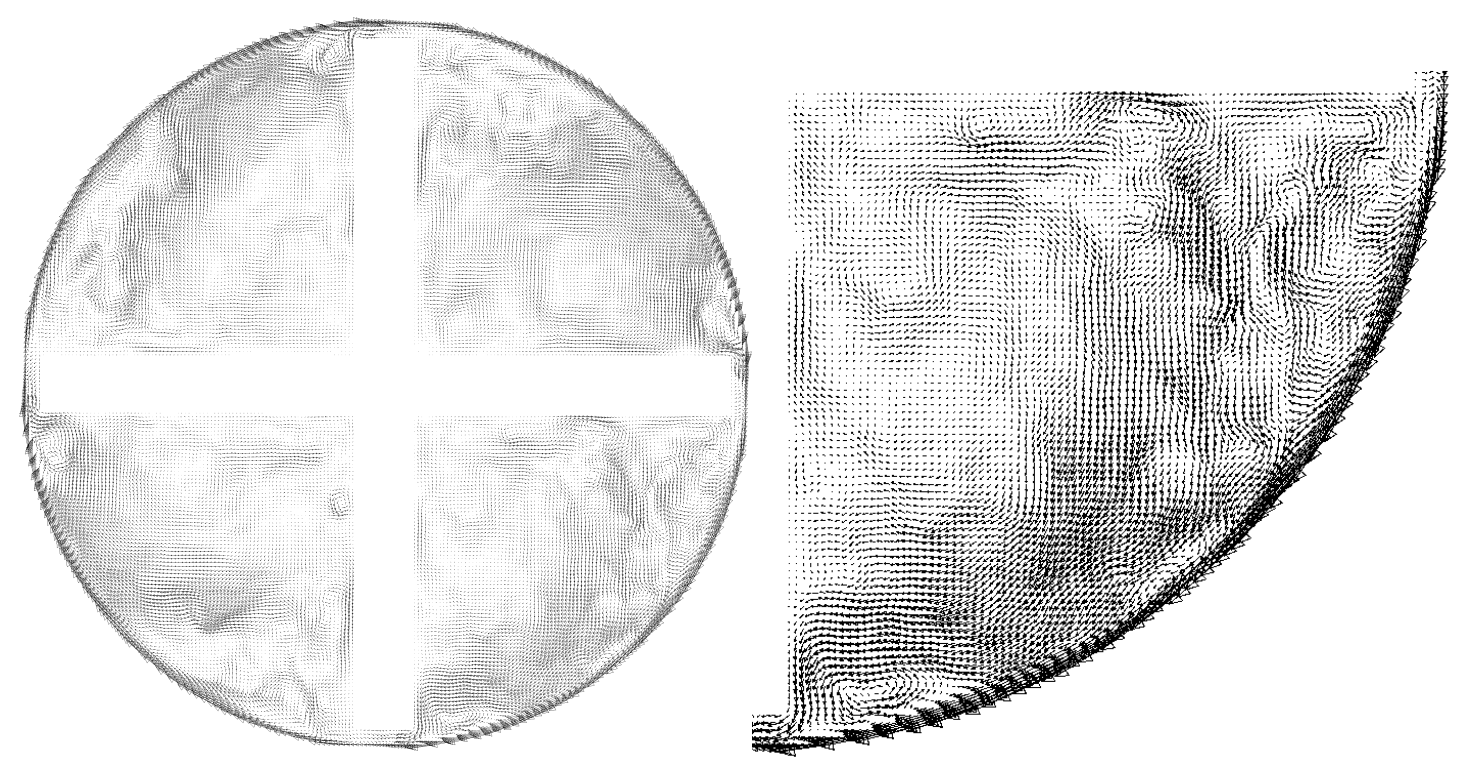

Figure 13. Instantaneous vertical view of the flow field in the frame of reference of the rotating scraper of the horizontal plane halfway the scraper height just above the $\mathrm{HE}$ and zoom of the right down quarter. The scraper rotates in the counter clockwise direction. In the rotating reference frame view of the figure, the wall now rotates clockwise. 


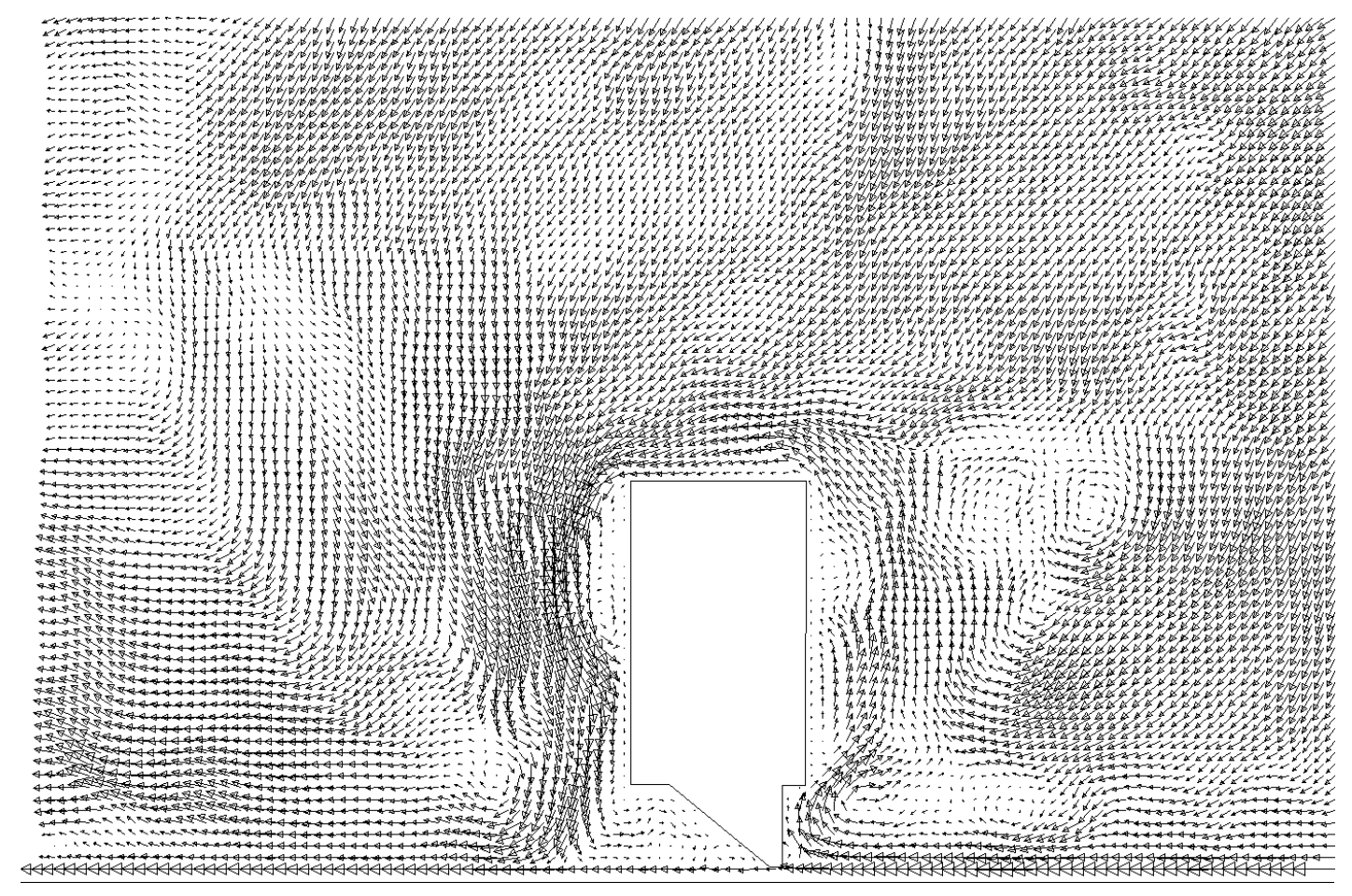

Figure 14. Instantaneous flow velocities of the vertical cut of the scraper region at the middle section of the scraper. 


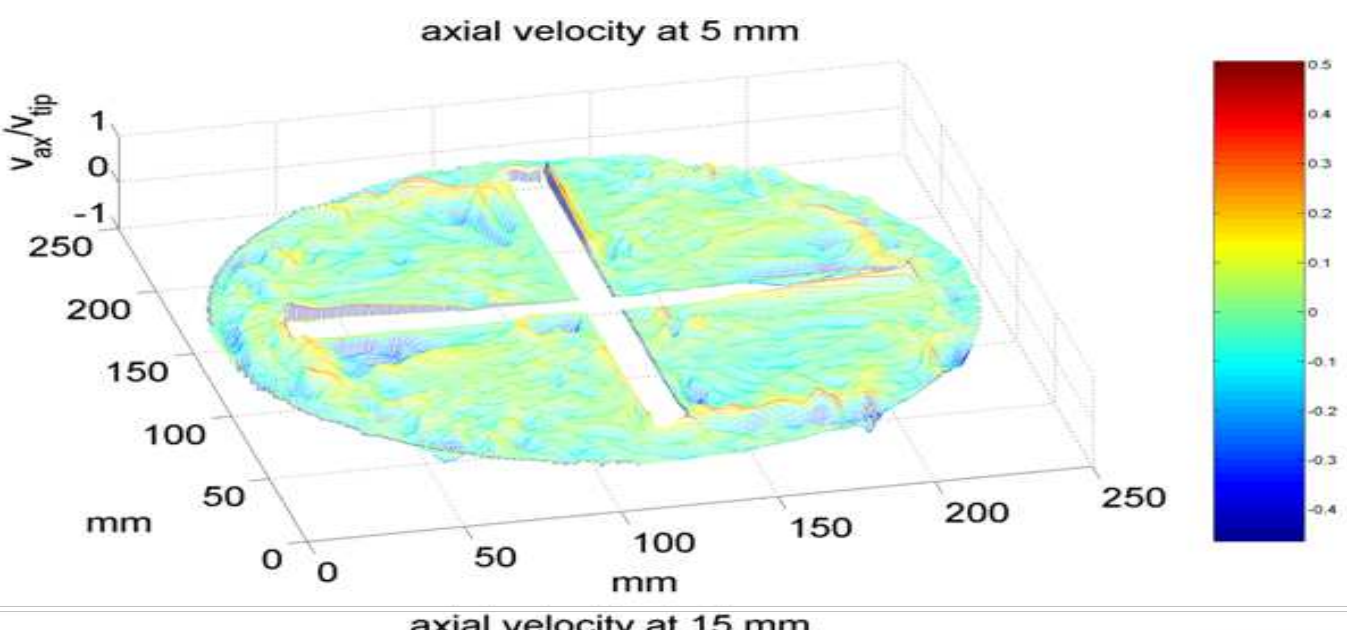

axial velocity at $15 \mathrm{~mm}$

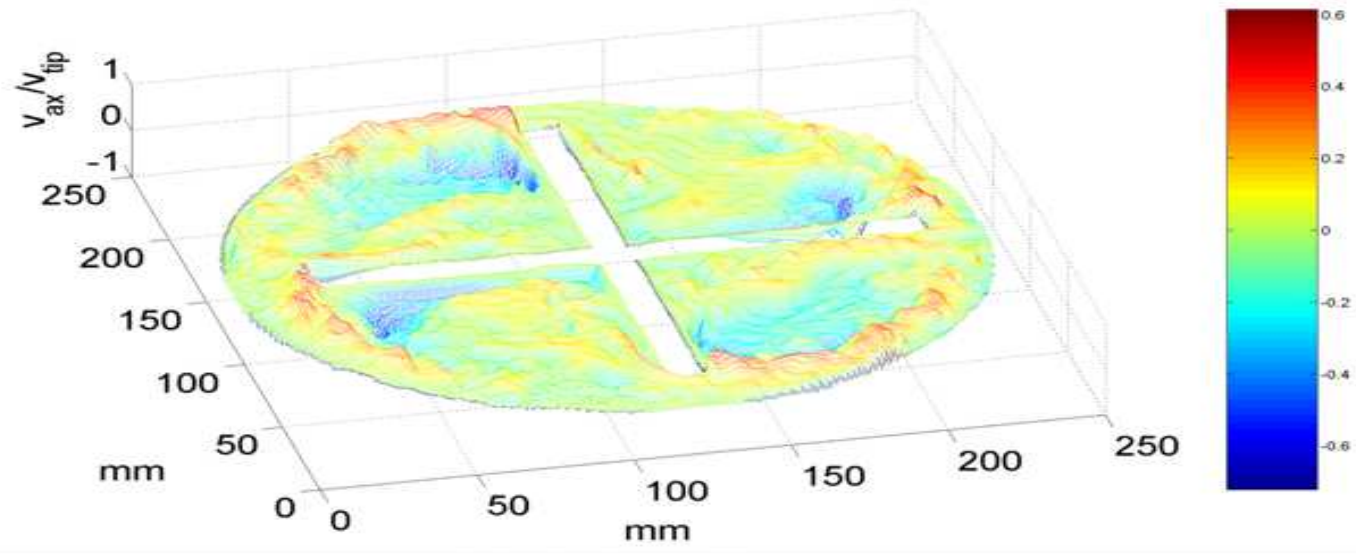

axial velocity at $30 \mathrm{~mm}$

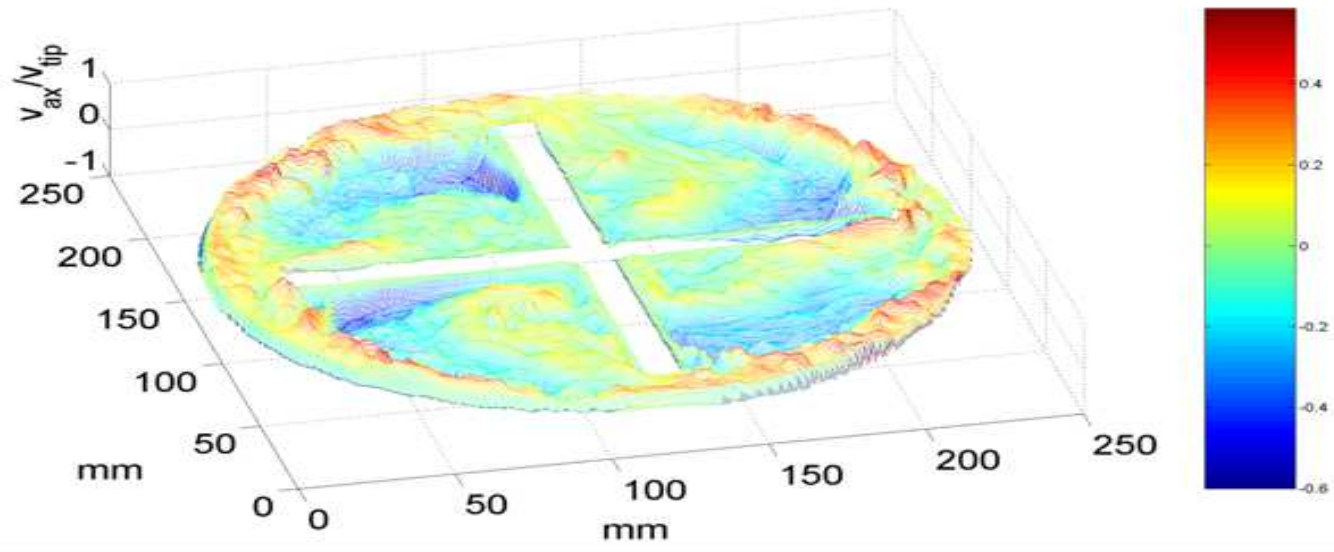

Figure 15. 3D surfaces of the instantaneous axial velocity at 5,15 and $30 \mathrm{~mm}$ from the heat exchanger surface. 